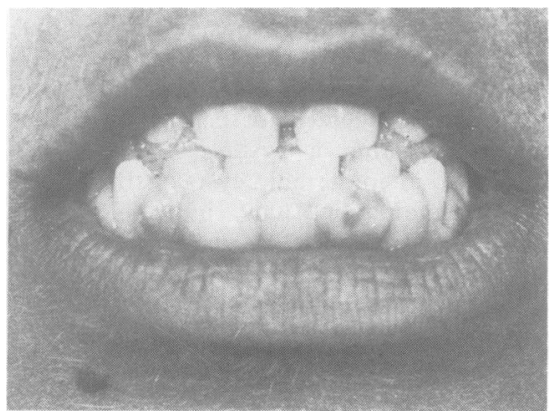

Figure Photograph showing gingival hyperplasia.

Electroencephalography showed frequent generalised bursts of polyspikes interposed on normal background activity. Eighteen months after starting sodium valproate therapy, gingival hyperplasia was noted which increased progressively. The gums were swollen, shiny, pale pink, firm and were covering approximately one half of the teeth. They did not bleed when touched and there were no engorged vessels (fig). The gingival hyperplasia was most prominent on the labial surface of the anterior teeth of the lower jaw. Investigations including haemogram, liver and renal function tests were again normal. Serum levels of valproate were not carried out. The drug regime was changed to a combination of carbamazepine $(600 \mathrm{mg}$ daily) and nitrazepam (15 $\mathrm{mg}$ daily) which resulted in poor control of seizures but a complete regression of gingival hyperplasia in three months.

This case appears to be only the second one of sodium valproate induced gingival hyperplasia. This patient differs from the earlier report of a 15 month old child with infantile spasms. $^{3}$

The patient belonged to an upper middle socio-economic group and was of average nutritional status without any evidence of nutritional deficiency, and she had never received diphenylhydantoin. She did not have any hormonal disturbances, was neither using oral contraceptive drugs nor was she pregnant and the fact that it regressed within three months of stopping sodium-valproate suggests that it is unrelated to puberty. That her gingival hyperplasia was not of an idiopathic hereditary variety is confirmed by the fact that there was no family history of gingival hyperplasia and that it regressed on stopping treatment with sodium valproate. Her blood counts were normal on two occasions and the gums were neither friable nor bled when touched which excluded the possibility of gum hyperplasia associated with leukaemia.

Several complications of sodium valproate are known ${ }^{4}$ but gingival hyperplasia is described only as a single case report in a 15 month old child with infantile spasm whose gingival biopsy showed oedematous connective tissue, dilated capillaries and dense infiltration by inflammatory cells especially mast cells and the authors implicated the role of mast cells in the pathogenesis of gingival hyperplasia due to sodium valproate. ${ }^{3}$ The effect of sodium valproate on the periodontal and oral health of epileptic patients (adults and children ${ }^{6}$ ) has been carried out in prospective studies which showed no unwanted effects on oral and dental health. Gingival hyperplasia is a common side effect of phenytoin therapy, but has also been observed with mephenytoin, ${ }^{7}$ and primidone and sulthiame ${ }^{8}$ therapy. The pathogenesis of gingival hyperplasia during phenytoin therapy may be due to stimulation of fibroblasts 9 or due to folic acid deficiency. ${ }^{10}$ There is no indication of the cause of the gingival hyperplasia in this patient.

M BEHARI Department of Neurology, All India Institute of Medical Sciences, New Delhi 110 029, India

1 Houck JC, Cheng RF, Waters MD. Diphenyl hydantoin: Effects on connective tissue and wound repair. In: Antiepileptic drugs, Woodbury DM, Penry JK and Schmidt RP, eds. New York: Raven Press, 1972:267-74.

2 Babcock JR. Incidence of gingival hyperplasia associated with dilantin therapy in a hospital population. J Amer Dent Assoc 1965;71: 1447-50.

3 Syrjanen SM, Syrjanen K. Hyperplastic gingivitis in a child receiving sodium valproate. Proc Finn Dent Soc 1979;75:95-98.

4 Dreifuss FE, Langer DH. Side effects of Valproate. Am Med 1988;88(Suppl IA):34-41.

5 Seymour RA, Smith DG, Turnbull DN. The effect of phenytoin and sodium valporate on the periodental health of adult epileptic patients. periodental health of adult epil
$J$ Clin Periodent 1985;12:413-9.

6 Eeg-Ollofsson O, Lundstrom A, Hamp SE. Oral state of children with epilepsy on treatment
stat state of children with epilepsy on treatment
with sodium valproate. Scand J Dent Res with sodium valpr

7 Babcock JR. Commiskey LV and White GO, Oral bilateral fibromas. Oral Surg 1966;21:4-8.

8 Stirrups DR, Inglis J. Tuberous sclerosis with non hydantoin gingival hyperplasia. Oral Surg Oral Med Oral Path 1980;49:211-3.

9 Hassel TM, Page RC, Narayanan AS, Cooper CG. Diphenyl hydantoin (Dilantin) gingival hyperplasia: Drug induced abnormality of connective tissue. Proc Natl Acad Sci, USA 1976;73:2902-12.

10 Vogel RI. Gingival hyperplasia and folic acid deficiency from anticonvulsant drug therapy: A theoretical relationship. $J$ Theoret Biol 1977; 67:269-78.

\section{A case of angiographically occult spinal} AVM

Intramedullary haemorrhage, or haematomyelia is not an unusual clinical symptom of a spinal arteriovenous malformation (AVM). Recent developments in neuroradiological methods usually identify the aetiology of haematomyelia preoperatively, but we report a case in which the AVM that was not identified by angiography.

A 40 year old woman was in good health until two days before admission, when while asleep she had an attack of sudden back pain on the right side radiating to the chest, and accompanied by vomiting. Next morning, she noticed weakness in her right leg and bilateral sensory loss below the thoracic level. Neurological examination on admission showed weakness in the right lowerextremity, increased bilateral patellar and ankle reflex with positive Babinski reflex, hypesthesia below left $\mathrm{T}-7$, hypalgesia and thermohypesthesia below right $T-7$, bilaterally diminished vibration sense below the thoracic cage and mild urinary disturbance. Haematological and coagulation tests were normal. The cerebrospinal fluid (CSF) was clear. Computed Tomography (CT) scan showed a haematomyelia as a high density area from the level of $\mathrm{T}-2$ to $\mathrm{T}-7$ with a "string-like" enhanced lesion at the T-4 level (fig 1). Myelography followed by a CT scan

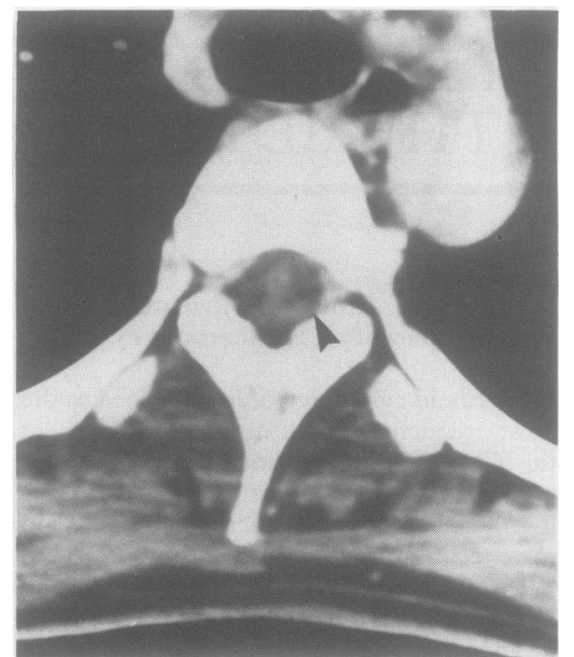

Figure 1 CT scan with intravenous contrast medium showing the haematomyelia and an intramedullary "string-like" enhanced lesion (arrow) at T-4 level.

showed cord swelling from the level of T -2 to T-4 without any abnormal vascular images. Magnetic Resonance Imaging (MRI) demonstrated a mixed intensity lesion at $T-3$ and $T-4$ level in both $T-1$ and $T-2$ weighted images. Complete spinal angiography was performed one week after admission. The right thyrocervical and costocervical trunk angiograms showed the anterior spinal artery, from the ascending cervical artery, to the $\mathrm{T}-4$ level without any intradural vascular malformation (fig 2). Other angiograms did not show abnormal findings. Her Adam-Kiewics artery branched from the left L-1 lumbar artery.

Four weeks after admission, we performed a laminectomy from $\mathrm{T}-2$ to $\mathrm{T}-5$ and made a small midline myelotomy at the $T-3 / 4$ level to evacuate the intramedullary haematoma, but did not examine the tissue surrounding the haematoma. Transient neurological improvement followed but then there was further neurological deterioration due to recurrent intramedullary bleeding, shown by CT scan two weeks after the first operation.

A second operation was performed about three weeks after the first operation. The midline myelotomy was extended and the intramedullary haematoma, surrounded by the fibrous tissue, was evacuated. Total removal of the haematoma and fibrous tissue revealed a tiny abnormal vascular lesion; it was completely removed and was shown by histological examination to be an AVM. The weakness of the right leg and the sensory disturbance had worsened and she was troubled by numbness of the right leg but she was able to walk with an aid of a stick three months after the second operation.

Although cavernous angiomas of the spinal cord are usually angiographically occult, ${ }^{4}$ we could not find a report of a case of haematomyelia caused by a spinal AVM that could not be identified by adequate spinal angiography. Only Veerpan's case ${ }^{5}$ of haematomyelia with a cryptic spinal AVM resembled our case, although in this case spinal angiography was not performed. In our case, it was difficult to determine the aetiology due to conflicting results of the neuroradiological examination, and the presence of AVM was not confirmed until the second operation. 


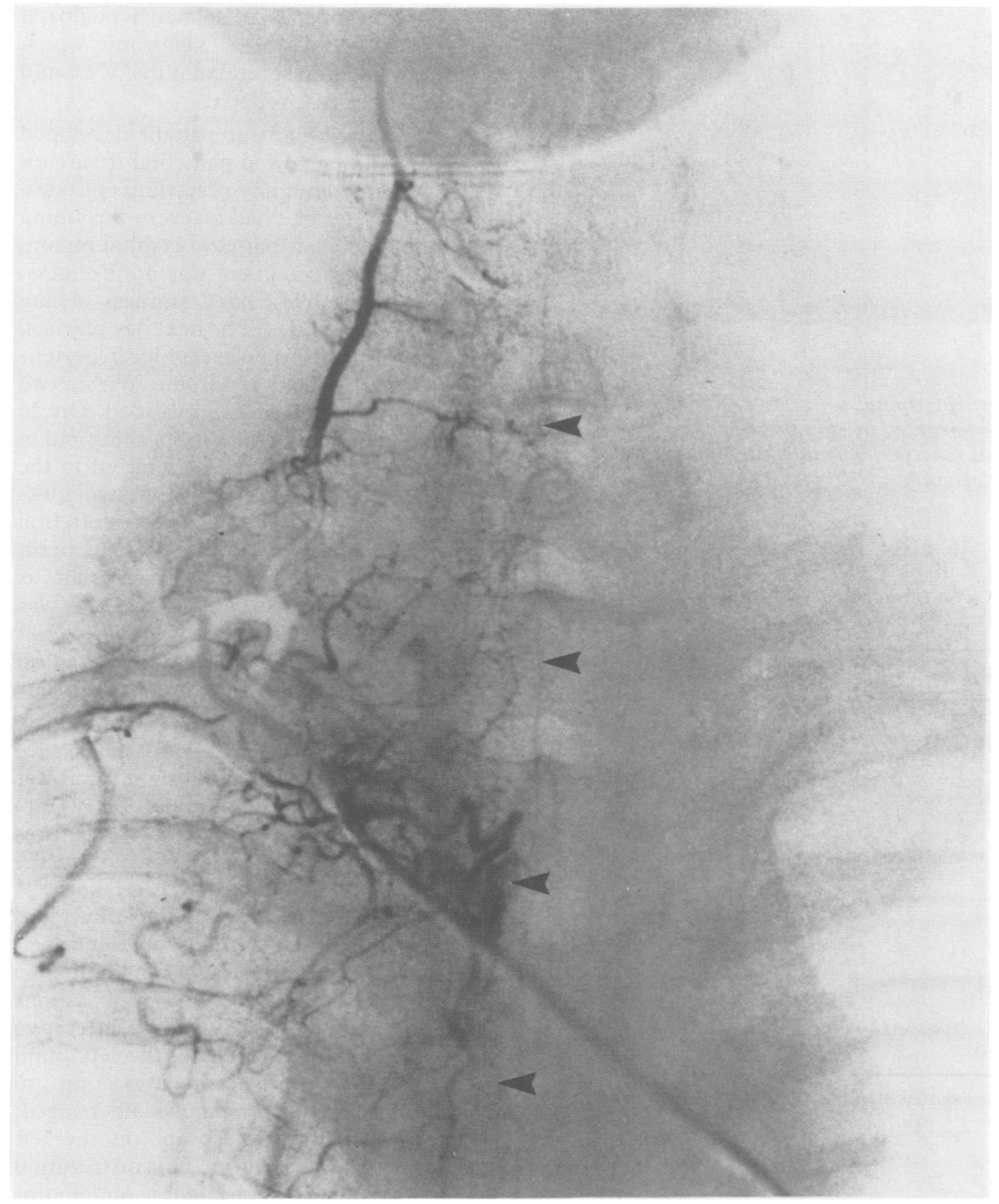

Figure 2 Right thyrocervical and costocervical trunk angiograms showing the anterior spinal artery (arrowheads), from the ascending cervical artery, to the T-4 level without any intradural vascular malformation.

Recently Lobato et $a l^{\beta}$ reported cases of angiographically occult intracranial vascular malformations and proposed several reasons why angiographic identification had not been possible. Their conclusions can also be applied to angiographically occult spinal vascular malformations. In our case the AVM was less than $5 \mathrm{~mm}$ in size and was located at the boundary of the territory between the two anterior spinal arteries, one from the deep cervical artery and the other from the AdamKiewicz's artery. We believe that its small size and location at the boundary of the anterior spinal artery territories are the most likely reasons why it was not recognised by angiography.

Smaller intracranial AVMs have a higher risk of haemorrhage, ${ }^{1}$ and we wonder if this can also be applied to spinal AVM lesions. Koos has suggested that a microangioma may be present in cases of haematomyelia in the absence of large spinal AVM. ${ }^{2}$ We suggest that bleeding due to a spinal AVM should be considered, even when angiography is negative.

TATSUYA ISHIKAWA KOUHEI ECHIZENYA HIROSHI MURAI MASAHARU SATOH Department of Neurosurgery Municipal Second Hospital,

Hokkaido, Japan

Correspondence to: Dr Ishikawa, Department of Surgical Neurology, Research Institute for Brain and Blood Vessels-Akita, 6-10, Senshu-Kubotamachi, Akita 010, Japan.

1 Forster DMC, Steiner L, Hakanson S Arteriovenous malformation of the brain. long-term clinical study. $J$ Neurosurg 1972;32:562-70.

2 Koos WT, Bock F. Spontaneous multiple intramedullary hemorrhages. $J$ Neurosur 1970;32:581-4

3 Lobato RD, Pertz C, Rivas JJ, Cordobes FC Clinical, radiological, and pathological spectrum of angiographically occult intracrania vascular malformation occult intracrania vascular malformation. Analysis of 21 cases 1988;68:581-3.

4 McCormick PC, Michelsen WJ, Post KD, Carmel PW, Stein BM. Cavernous malformations of the spinal cord. Neurosurgery tions of the

5 Veerapen RJ, Sbeih IA, O'Laoire SA. Surgical treatment of cryptic AVM's and associated treatment of cryptic AVM's and associated
hematoma in the brain stem and spinal cord. hematoma in the brain stem
$J$ Neurosurg 1986;65:188-93.

Extraneural metastases from brainstem astrocytoma through ventriculoperitoneal shunt

In 1954 Wolf et al $^{1}$ reported a case of a 14 year old female with a thalamic glioblastoma who needed a ventriculo-pleural shunt to relieve hydrocephalus. She died 8.5 months after shunt placement, and at necropsy tumoural cells were found in the pleural cavity. There have been at least 43 other cases of CNS primary tumours that have metastasised through shunts used in the treatment of hydrocephalus. We report the case of a patient who had a brainstem astrocytoma with extraneural metastases through a ventriculoperitoneal shunt (VPS) with development of ascites, and spinal cord seeding.

A four year old girl, previously in good health was admitted to hospital on 27 December, 1985 with a clinical picture of continuous and intense occipital headache, dizziness and vomiting. Her past medical history was normal. Positive neurological findings included vertical nystagmus in upward gaze and horizontal nystagmus in right gaze; palsy of the VI, VII and IX left cranial nerves; right crural paresis with hypertonia, hyperreflexia and Babinski sign; right limb dysmetria and ataxic gait. A brain CT revealed a left pontomesencephalic hypodense area extending to the left cerebellar peduncle with an irregular enhancing after injection of contrast material. The fourth ventricle appeared displaced backwards with a mild compression in its floor There was also a moderate supratentorial hydrocephalus. The patient improved considerably after treatment with dexametasone and radiotherapy. Five months after diagnosis she was again admitted because of bilateral action tremor, dysmetria and ataxic gait. A month later she experienced headache and vomiting, and was found to have bilateral papilloedema in addition to the previous neurological signs.

A second brain CT showed a prominent obstructive hydrocephalus. A VPS was implanted and the patient's symptoms rapidly improved. Ten months after diagnosis she complained of a sudden intense back pain and developed a progressive ascending hypotonic quadriparesis with arreflexia and bilateral Babinski sign. Sensory loss and sphincter dysfunction were not present. A new CT showed brainstem widening and cystic lesions in the left pontocerebellar angle, retrocerebellar region and right medial temporal lobe, that were interpreted as necrotic cysts secondary to radiotherapy. Eleven months after diagnosis, and five months after VPS placement she presented with abdominal distension caused by ascites.

An abdominal ultrasound failed to show masses or encapsulated collections. On 7 November, 1986 the abdominal catheter was removed, but the ascites followed a progressive course. Two cerebrospinal fluid (CSF) specimens showed moderate rise in protein content, but all CSF cytologies were negative. Ascitic fluid had normal biochemical parameters and negative cytology. Because of the poor condition of the patient we decided to avoid aggressive diagnostic and therapeutic manoeuvres. She died two weeks later.

Necropsy showed a white mass in the left pontomesencephalic region spreading to the cerebellar peduncles, septum, fourth ventricle walls and periventricular white matter. There was also involvement of the arachnoid of the cerebellum and the whole length of the spinal cord but with sparing of the spinal cord itself and the spinal nerve roots (fig $A$ ). Peritoneum was diffusely thickened, white in colour and nodular in appearance. Histological examination showed a diffuse astrocytic growth in the CNS but with sparing of the abdominal organs. Glial fibrillar astrocytic protein (GFAP) stain was strongly positive for samples of the tumour from brainstem, 